\title{
LA INVESTIGACIÓN BIOMÉDICA ACTUAL: UN CUESTIONAMIENTOA LA SOCIEDAD EN SU CONJUNTO
}

\author{
María Lucrecia Rovaletti*
}

\begin{abstract}
Resumen: La segunda revolución biomédica pone en cuestión a la sociedad humana en su totalidad en la medida que le otorga al hombre el dominio de tres sistemas fundamentales: el de la reproducción, el de la genética y el del sistema nervioso. Los nuevos ensayos experimentales sobrepasan la protección o el restablecimiento de la salud y llevan a cuestionar las nuevas técnicas en tanto son capaces de modificar la evolución de las especies, más aún, de modificar la humanidad en tanto que especie.

No se pretende postular un programa simplemente de control de la "ciencia", ni alimentar sospechas recíprocas generando una interminable querella sobre el progreso científico. Para avanzar hacia una real solución del problema y una cooperación entre la cultura científica y los valores humanos, es necesario transformar la experimentación sobre el hombre en experimentación con el hombre. ¿En qué medida la investigación médica puede considerar nuevas alianzas entre las tareas científicas y las demandas humanas? Urge plantear una "comunidad científica ampliada" que permita a los nuevos actores sociales una apropiación colectiva de los saberes.
\end{abstract}

Palabras clave: Biomedicina, investigación

\section{BIOMEDICAL RESEARCH NOWADAYS: QUESTIONING SOCIETY AS A WHOLE}

Abstract: The second biomedical revolution puts into question human society as a whole inasmuch as it gives to human being the dominion over three fundamental systems, namely: reproduction, genetics, and the nervous system. Current experiment assays go beyond either health protection or health recovery, thus allowing new techniques to be challenged in the sense that they may modify the evolution of species; furthermore they may modify humans as a species.

On no account, however, are we suggesting simply that some control program on science be implemented, nor are we willing to sow mutual suspicions likely to generate an endless quarrel on scientific advance. To progress towards an actual problem solving process coupled to a cooperation between scientific culture and human values, it is necessary that research on people must be transformed into researching with people. To which extent, however, medical research is in a position to envision new alliances between scientific tasks and human demands? Setting up an expanded scientific community able and willing to present new social actors with a collective appropriation of knowledge is an urgent commitment to be considered thoroughly.

Key words: Biomedicine, research

\section{A PESQUISA BIOMÉDICA ATUAL: UM QUESTIONAMENTO À SOCIEDADE NO SEU CONJUNTO}

Resumo: A segunda revolução biomédica coloca em questão a sociedade humana em sua totalidade na medida em que outorga ao homem o domínio de três sistemas fundamentais, o da reprodução, o da genética e o do sistema nervoso. Os novos ensaio experimentais vão além da proteção ou do restabelecimento da saúde e levam a questionar as novas técnicas enquanto são capazes de modificar a evolução das espécies, ainda mais, de modificar a humanidade enquanto espécie. Não se pretende postular um programa simplesmente de controle da "ciência", nem de alimentar dúvidas recíprocas criando uma interminável discussão a respeito do progresso científico. Para avançar em direção de uma real solução do problema e uma cooperação entre a cultura científica e dos valores humanos, é necessário transformar a pesquisa sobre o homem em pesquisa com o homem. Em que medida a pesquisa médica pode considerar novas alianças entre as tarefas científicas e as demandas humanas? Urge criar uma "comunidade científica ampliada" que permita aos novos atores sociais uma apropriação coletiva dos saberes.

Palavras chave: Biomedicina, pesquisa

* Investigador Independiente del CONICET. Docente de Antropología Médica y de Bioética de la Facultad de Medicina. Profesora Regular del Psicología Fenomenológica de la Facultad de Psicología. Universidad de Buenos Aires.

Correspondencia: mlrova@psi.uba.ar 
“... precisamente porque todo el mundo -terapeutas, investigadores y ciudadanos en general- está ávido de descubrimientos cientificos sensacionales, básicos o aplicados, es que quizá se impone una reflexión ética profunda sobre la investigación biológica, y correlativamente, un debate público, en la medida que toda admiración ante la ciencia en curso y un cierto acostumbramiento a todas las audacias técnicas tienden a embotar el umbral individual y colectivo de la interrogación ética." (CCNE, en Michaud, 1990, p. 8)

En una entrevista para el libro "Le temps de la responsabilité", Jean Bernard señala las dos grandes revoluciones biológicas que nos ha tocado vivir en el reciente siglo XX. La primera, la revolución terapéutica, que comienza hacia el año 1937 con las sulfamidas y que permitió al hombre triunfar sobre enfermedades hasta entonces incurables como la tuberculosis, la sífilis, las grandes septicemias, y los grandes desórdenes químicos del humor. La segunda comienza 20 años más tarde, con una fuerte dirección cognitiva. Mientras la primera era empírica, esta última se presenta como racional, metodológicamente rigurosa, y es la que ha permitido el desarrollo de la biología molecular y los descubrimientos genéticos. La actual "investigación biomédica" se dirige al conocimiento no sólo del hombre enfermo en una búsqueda del beneficio directo para su salud, sino también al hombre sano ya sea en un ambiente ordinario, artificial o extremo.

En efecto, la profesión médica, independientemente del deber de aliviar el sufrimiento de los enfermos y de sus familias, tiene como exigencia también la investigación ya que ello, tarde o temprano, redundará en utilidad para la salud de la humanidad. De allí que el Comité Nacional de Ética de Francia, en el año 1986, considerara que la medicina tiene que ser necesariamente experimental, traduciendo con ello la idea de una obligación por parte del cuer- po médico de investigar. Si bien es cierto que, en ciertos casos, hay un beneficio directo a determinado tipo de enfermo, a menudo el estudio de individuos sanos se justifica desde una motivación altruista, en tanto puede servir con posterioridad para otros: es el pasaje de la ética centrada en el bien del individuo enfermo a una ética de la solidaridad centrada en el bien colectivo.

\section{¿El progreso de la ciencia o la ideología del progreso científico?}

Si la primera revolución biomédica tenía estrecha relación con el ejercicio de la medicina, en particular respecto a los ensayos farmacológicos y el tratamiento de los pacientes, la segunda pone en cuestión a la sociedad humana en su totalidad, en la medida que le otorga al hombre el dominio de tres sistemas fundamentales: el de la reproducción -a través de las áreas de la contracepción, la inseminación artificial y fecundación in vitro-, el de la genética o dominio de la herencia y el del sistema nervioso.

Estas nuevas experimentaciones que provienen de las ciencias de la vida, en tanto son capaces de modificar no sólo la evolución de las especies sino la humanidad misma en tanto especie, serán puestas en cuestión por los planteos bioéticos: la naturaleza globalmente se encuentra amenazada por la intervención del hombre en la medida que puede llegar a afectar a los grandes equilibrios del cosmos. Por ello, "en todas partes los ciudadanos, directamente implicados, exigen a los responsables y esperan sanciones penales contra estos últimos en caso de catástrofes", insiste Puytorac.

A pesar de ello, todo un cientificismo fundado en una férrea confianza en la ciencia actual la juzga como una honra del espíritu humano y un factor de progreso. Aunque se reconozcan los riesgos y peligros que ella conlleva, 
se los acepta como el precio que hay que pagar para el avance del saber. ¿Cómo es posible entonces mantener un equilibrio entre la libertad y/o la obligación del conocimiento y el bien público? (Jonas, 1997, p. 65). El problema ético y filosófico se coloca exactamente en este punto. Precisamente,

"Cuando se comienza a tratar al hombre según los criterios de la cientificidad 'objetiva' [...] se transforma efectivamente al ser humano en objeto y como consecuencia se lo suprime en tanto que ser humano"(Ladrière, 1988, p. 289).

Se asiste, por otra parte, a un cambio cualitativo de la ciencia por acumulación de los efectos cuantitativos del progreso técnico. La investigación, más que orientarse al conocimiento, se dirige ahora a la producción; y la búsqueda de conocer se reduce, a menudo, a una coartada ante la voluntad de dominio técnico, frente al cual la salud cuenta cada vez menos. Si en la modernidad se podía hablar de la ciencia como una Bildung (construcción) (Heidegger), en la postmodernidad ésta se ha convertido en una empresa, manejada con la lógica de la eficiencia y ordenada a una lógica del lucro. La tecnociencia ha devenido ahora una empresa para la empresa.

El productivismo pone a nuestra disposición, de modo directo o encubierto, una serie de artificios y tecnologías, sin que nosotros podamos verificar si son necesarios para vivir mejor o para sufrir menos y sin que podamos cuestionar la ganancia o la pérdida social que resulta de ellos. Más allá de las simples necesidades, se demanda a la ciencia y a la sociedad que atienda los deseos más fantasmáticos, que nunca logran ser satisfechos.

La creciente dependencia de los individuos en relación con las técnicas subraya la responsabilidad del investigador que ha contribuido ampliamente a ellas. El investigador podrá reflexionar sobre la fundamentación ética del trabajo o plantear una moratoria o tomar la decisión de limitar su campo de investigación. En este sentido, el "J'arrête" (me detengo) del científico francés Jacques Testart en 1986 constituye una querella en el seno mismo de este "progreso indefinido". Pero nadie puede estar seguro de que alguien menos escrupuloso no continúe la tarea posteriormente, pues, como bien se sabe, el "pavor" pronto se convierte en un sentimiento pasajero que acaba cediendo lentamente ante la atracción permanente del artificio utilitario o caprichoso.

En este sentido, a los Comités de Ética se les ha otorgado -entre otras- una función de aseguramiento y ellos mismos reconocen que buscan restaurar la confianza a veces perdida en la comunidad científica. Por eso, en sus recomendaciones y en sus elecciones éticas buscan beneficiar a los investigadores, pero siempre en el marco de compromisos socialmente aceptados. No obstante, algunos hasta cuestionan a los mismos Comités de Ética Científica, en la medida en que están compuestos por investigadores que son juez y parte, y aunque éstos lograran limitar algunas investigaciones tampoco hay certeza de que los laboratorios necesariamente cumplan con ello.

Por su parte, las Comisiones Nacionales de Bioética tratan de entablar un acuerdo en la discordia y, cuando ello se torna imposible, se ven obligadas a marcar las fronteras de lo aceptable, en función de la regla del menor mal o costo ético. Contribuyen de este modo a proponer los medios para que la búsqueda del saber científico acuerde con los valores comunes de una sociedad organizada gracias a un entendimiento recíproco. Precisamente, "la novedad de la época reside, quizá, en esta instauración de un proceso colegiado que predispone moralmente al proyecto colectivo de la ciencia" (Langlois, 1992, p. 158). 
Aunque la mayor parte del público no tenga información detallada acerca de las discusiones científicas ni de las disposiciones legales, la sociedad, sin embargo, no puede dejar de interrogarse sobre el valor intrínseco de esta revolución cognitiva en el campo de la biomedicina. Asistimos a la mutación de prácticas individuales en prácticas institucionales, y esto no sólo concierne a los individuos como responsables de estos actos, sino a toda la sociedad que está confrontada con elecciones de las que depende el futuro de la humanidad.

\section{¿Control social de las biociencias o comunidad científica ampliada?}

Estos análisis sin embargo no pretenden postular un programa simplemente de control de la "ciencia" o, en su acepción más común, de "sus actividades científicas", pues ello sólo conduce a una vigilancia externa y polémica y alimenta sospechas recíprocas, generando una interminable querella sobre el progreso, sin superación fructífera alguna. Como dice L. Sève, la biociencia no dejará de ser in-ética por compulsión ni devendrá ética por conminación. Se trata no de un freno ético, en oposición a la dinámica científica, sino del tenaz esfuerzo por integrar la dimensión ética a la dinámica científica, como insiste el Comité Nacional de Ética Clínica de Francia (Michaud, 1990, p. 49). No se podrá avanzar hacia una real solución del problema si no se trabaja en una aproximación y una cooperación entre la cultura científica y los valores humanos, donde ambas partes tienen mucho que aprender.

¿No sería mejor transformar la experimentación sobre el hombre en experimentación con el hombre? (Langlois, 1992). Los hombres estudiados dejarían de ser simples "objetos" pasivos de una dirección cognitiva reductora para convertirse en "sujetos" activos del esfuerzo de conocimiento que les concierne. Ya no se trata de dar un informe esclarecido al sujeto con el solo objetivo de lograr un consentimiento, sino de asociarlo a los fines de la investigación y a los procedimientos correspondientes. Se trata de pasar de "voluntario pago" a "colaborador científico".

Este cambio epistemológico de fundamental importancia ya fue planteado en la metodología de las ciencias sociales a través de un principio de incertidumbre análogo al de Heisenberg, según el cual el observador participa de la observación y, a su vez, es influido por ello al mismo tiempo que observa. ¿En qué medida la investigación médica puede asumir esta renovación?

Actualmente, existe una cooperación para elaborar protocolos experimentales entre investigadores y familiares de enfermos graves o crónicos, nucleados en general en asociaciones de autoayuda. De este modo, al aproximarse los científicos a esta "construcción profana de la enfermedad", les es permitido participar de este otro punto de vista para comprender la particular situación de los enfermos: la perspectiva profana se transforma en una perspectiva cognitiva.

Del mismo modo, desde 1996 el Welsh Institute for Health and Social Care (WIHSC), en Inglaterra, está explorando las perspectivas públicas en genética y atención de salud. En efecto, basados en el modelo legal de juicios, un Jurado de Ciudadanos, durante un período de 4 ó 5 días, examina el testimonio de expertos, delibera sobre una cuestión o una serie de cuestiones y presenta las conclusiones. Estos jurados están constituidos por personas legas, en su calidad de ciudadanos, a diferencia de la de usuarios de servicios, consumidores o miembros de grupos con intereses específicos. Se parte de la premisa de que la gente común -con el tiempo suficiente, el apoyo, los recursos y la oportunidad- es capaz de arribar a decisiones en temas políticos de complejidad. 
De allí que, en las ciencias de la vida, el "conocimiento científico" no puede estar integrado solamente por un sistema conceptual (Popper) o mundo objetivo de enunciados, teorías, problemas, argumentos, libros y revistas, en tanto contenidos de sentido lógico, sino además por un sistema social, constituido por la comunidad científica (Kuhn) donde se realiza el proceso de investigación.

Es verdad que los actos fundamentales de la práctica de la salud constituyen un proceso de definición social fuertemente controlado por la profesión médica, en tanto organización social legitimada por cada cultura a través de sus normas (Freidson), y que son los mismos individuos los que entregan ese control para satisfacer la necesidad de ser cuidados y protegidos contra fuerzas internas que parecen, a veces, presentarse como potencialmente abrumadoras.

Sin embargo, actualmente la sociedad requiere de nuevas alianzas entre las tareas científicas y las demandas humanas, que permitan plantear una "comunidad científica ampliada" donde sea posible una apropiación colectiva de los saberes por parte de nuevos actores sociales.

Desde el punto de vista de la construcción social del conocimiento, se trata de abordar un nuevo nivel de complejidad donde pueda englobarse al observador y al observado en sus necesidades y en sus responsabilidades El equipo investigador forma parte del conjunto social, no ya como depositario del saber, sino como facilitador al que la sociedad encarga que libere las posibilidades cognitivas y terapéuticas presentes en el mismo sistema. Dado que la interacción entre los sistemas profesionales $\mathrm{y}$ profanos funciona como un mecanismo de retroalimentación, todo cambio en un nivel del sistema producirá una alteración simultánea en los otros. Ese punto de vista sólo es posible desde una actitud metodológica y operativa que integre la participación de la comunidad, desde los momentos decisionales hasta la evaluación de las acciones. Ninguna comunidad puede enfrentar una crisis si no apela al fortalecimiento de su organización y, por tanto, de la solidaridad. La ética no se reduce a una disciplina teórica sino que constituye un elemento esencial de la praxis social.

Frente a un concepto de "responsabilidad", en cuanto imputabilidad por las acciones del pasado, Jonas postula para la era tecnológica un "principio de responsabilidad" que se orienta al futuro más allá de las consecuencias previsibles, y que le cabe no sólo a los investigadores, sino a la sociedad en su conjunto.

La participación en los Comités de Ética de los distintos agentes del campo de la salud, de distintas disciplinas, como derecho, antropología, filosofía, etc., de distintas religiones, de organizaciones no gubernamentales y de simples ciudadanos constituye un paso importante.

Faltaría preguntarnos, ¿en qué medida los conjuntos sociales ${ }^{1}$, más cerca del paternalismo y del participacionismo subordinado, pueden y quieren dejar de ser considerados meramente como "pacientes" y devenir agentes de promoción cognitiva, colaboradores activos? Estos antiguos mecanismos de transacción social ${ }^{2}$ son tributarios no sólo de los profesionales de la salud, sino también del público consumidor de la oferta biomédica que se presenta como

Los "conjuntos sociales" o humanos constituyen "un sistema de estructuración" en el cual los individuos adquieren una significación mediante los mensajes (verbales, afectivos, corporales, económicos...) que intercambian en un contexto institucional; por tanto por medio de sus diferencias (Hochmann). Cfr. Rovaletti 1990.

2 Se denominan "procesos transaccionales" (E. Menéndez) a los procesos de apropiación que surgen de la integración social, los cuales deben ser analizados en cada situación histórica determinada. Ahora bien, que dichos procesos sean unilaterales, de subordinación o no, dependerá de la estructura social que se haga cargo de los mismos. Cfr. Rovaletti 1990. 
expresión de una ciencia pretendidamente omnisciente, ante la cual cabe una sumisión pasiva. Precisamente la secularización ha transferido a la ciencia, en particular a la medicina, la responsabilidad de enfrentar toda una serie de males sociales.

Por ello, estas "apropiaciones" deben constituir no sólo mecanismos válidos en orden a las "estrategias del saber", sino también respecto a las "evaluaciones éticas" de las investigaciones experimentales. Esto permitiría además enriquecer el principio de autonomía y de solidaridad responsable al ser asumido y apropiado por "los conjuntos sociales".
Los cambios realmente sustanciales suelen ser dolorosos, porque obligan a encontrar nuevos equilibrios, pero nos colocan en ese camino sinuoso, imprescindible y no predeterminado por el cual nos hemos convertido en la especie homo sapiens. Por eso, no se puede ni se debe renunciar a tales avances, ni sorprenderse por las dificultades que se puedan plantear socialmente.

La ciencia adquiere vida cuando es objeto de debate público, cuando la sociedad se plantea el poner de acuerdo lo que desea y lo que es posible; cuando surge la cuestión si es aconsejable poner limites a la ciencia, al igual que en cualquier otra actividad (Sanchez Ron, 1999, p. 68).

\section{Bibliografía}

Arboleda Flores J, Weisstub D. Poblaciones vulnerables: protección de su condición como sujetos de investigación. La Plata: Quirón; 1996: 54-74.

Berlinguer G. Ética de la Salud. Buenos Aires: Editorial Lugar; 1994.

CIOMS /OMS: Normas éticas internacionales para las investigaciones biomédicas en sujetos humanos. Cuadernos del Programa Regional de Bioética 1996; 3: 126-140.

Escudé Casals JM. Una ética para la era tecnológica. Cuadernos del Programa Regional de Bioética 1997; 5: 63-84.

Hitzenberger G. La experimentación en el hombre. Monografias. Cuadernos de Bioética 1996; 4: 425-431.

Hottois G. Essais de philosophie bioéthique et biopolitique. Paris: Vrin; 1999.

Hottois G, éd. Aux fondements d'une éthique contemporaine. H. Jonas et H.T. Engelhardt. Paris: Vrin; 1993.

Hottois G. Evaluer la technique, Paris, Vrin, 1988

Jonas H. Le principe responsabilité. Paris: Du Cerf ; 1990.

Jonas H. Técnica, medicina y ética. La práctica del principio de responsabilidad. Barcelona: Paidós; 1997.

Kottow M. Investigación en seres humanos: Principios éticos internacionales. Cuadernos del Programa Regional de Bioética 1996; 3: 42-54.

Ladrière J. Expérimentation humaine et droits de l'homme. En: Yotopoulos-Marangopoulos A. Expérimentation biomédicale et droits de l'homme. Paris: PUF; 1988.

Ladrière J. Les enjeux de la rationalité. Le défi de la science et de la technologie aux cultures. Paris: Aubier-UNESCO; 1977. 
Langlois A. Éthique et connaissance; la recherche à visée cognitive. En : Parizeau MH, ed. Les fondements de la bioéthique. Bruxelles: De Boeck Universität; 1992: 153-168.

Lecourt D. Contre la peur. Paris: Hachette; 1990.

Lenoir F. Le temps de la responsabilité. Entretiens sur l'éthique. Paris: Fayard; 1991.

Memmi D. Les gardiens du corps. Dix ans de magistère bioéthique. Paris: Éditions de L'École des Hautes Études en Sciences Sociales; 1996.

Michaud J, comp. Éthique et connaissanse. Una réflexion sur l'éthique de la recherche biomédicale. Paris: La Documentation française; 1990.

Puytorac P. De la biophilosophie à une éthique de la biologie: la societéface à la biologie. Paris: L'Harmattan; 1998

Queré F. L'éthique et la vie. París: Odile Jacob; 1991.

Rovaletti ML. On cosmic Responsibility. Concordia. International Zeitschrift für Philosophie 1998; 33: 77-86.

Rovaletti ML. El saber biomédico y la metáfora mecanicista. Perspectivas Bioéticas en las Américas 1998; 6: 24-39.

Rovaletti ML. Enfermedad y tejido social. Relaciones 1990; 78.

Salas S. Estructura y función de los comités de ética de la investigación clínica. Cuadernos del Programa Regional de Bioética 1996; 3: 94-110.

Sanchez Ron JM. Un nuevo mundo científico. Revista de Occidente 1999; 214: 65-68. 